\title{
Investigation of Optimum TTL Threshold value for Route Discovery in AODV
}

\author{
Puneet Bindra \\ M.tech Scholar \\ SBSSTC, Ferozepur
}

\author{
Jaswinder Kaur \\ A.P (ECE) \\ SBSSTC, Ferozepur
}

\author{
Gurjeevan Singh \\ DIC (ECE) \\ SBSSTC(Polywing), Ferozepur
}

\begin{abstract}
In the mobile Ad hoc networking paradigm there is no fixed infrastructure and packets are delivered to their destinations through wireless multi hop connectivity. MANETs are in general characterized by mobile nodes with limited energy, constrained computing capability and absence of any base stations. Mobile nodes undertake role of routers engaging in some routing protocol required for deciding and maintaining the routes. MANET allows unrestricted mobility of the enlisted terminals as long as the least one terminal is within transmission range. Transmission range of wireless Adhoc network is restricted by its power consumption due to limited battery power of a wireless node. Reactive routing protocols are favoured in MANET's because they help in reducing overheads by continuously sending the data for better communication. These protocols suffer due to route discovery process. We focus on the problem of finding best search set of TTL(Time to live) values for expanding ring search to improve quality of service and evaluate performance of AODV by varying number of mobile nodes and nodes speed. Expanding ring search based on TTL in terms of early or delay in network flooding cause quality of service to fluctuate randomly. In this paper, early or delay in network flooding is employed to search for destination in a region limited by time to live (TTL). The performance of such expanding ring search schemes depends largely on TTL search sets. It is simulated using OPNET 14.5 simulator.
\end{abstract}

\section{Keywords}

MANET, OPNET 14.5, Routing Protocol

\section{INTRODUCTION}

MANETs are generating a lot of interests due to $3 \mathrm{G}$ and $4 \mathrm{G}$ activities. Mobile and portable nature of MANET's increased the popularity of these networks. A mobile Ad hoc network is a collection of wireless mobile hosts that dynamically create a temporary network without fixed infrastructure. They created new set of demands to be implemented and to provide efficient better end to end communication [1]. MANET's can be setup anywhere anytime without using any pre-existing network infrastructure [2]. Links are created and destroyed in an unpredictable way in MANETs which makes quite challenging the determination of routes between each pair of nodes that want to communicate with each other. A variety of routing protocols for ad hoc networks have been proposed in recent past. Proactive protocols disseminate routing information from each node to each other periodically and find routes continuously whereas reactive protocols find routes on demand. Reactive or on demand ad hoc routing protocols typically make use of request query broadcast to discover routes when a source to destination communication is needed.
AODV is one of the most representative reactive routing protocol. AODV is hop by hop routing protocol which introduces a more dynamic strategy to discover and repair route when compared to other on demand protocols [3]. In AODV, the network is silent until a connection is needed and route is created only when requested by a network connection and information regarding this route is stored only in the routing tables of those nodes that are present in the path of the route [4]. AODV maintains only active routes to reduce overheads and control traffic. At that point the network node that needs a connection broadcast a request for connection. It uses different messages to discover and maintain links- Route Request (RREQ), Route Reply (RREP) and Route Error (RRER). These messages type are received via UDP, the normal IP header processing applies. When the source node desires to establish communication session, it initiates a path discovery process by broadcasting route request (RREQ) message to locate the other node. Next the route request is forwarded by intermediate nodes which also create a reverse route for itself to reach the destination. When the route request reaches the node with route to destination it creates route reply (RREP) which contains the number of hops that are required to reach the destination. The route created by each node from source to destination is a hop by hop state and not the entire route as in source routing. Expanding ring search is a prominent technique used for route discovery in mobile ad hoc networks. In this paper, we model TTL based expanding ring search for reliable and improved quality of service and evaluating performance of AODV by varying number of mobile nodes and node speed. The paper is structured as follows: a brief overview of the expanding ring search is given in Section 2. Section 3 then enfolds Experimental set up and Results are displayed in section 4 .Section 5 concludes this work and gives an outlook to further possible developments and research topics in this area.

\section{Expanding Ring Search}

In AODV protocol, the route is built on demand and is not updated until the route breaks or times out. The route can't adapt to topology changes and breaks frequently in the case of high mobility. AODV uses local repair to restrict the route discovery zone so as to reduce overheads and improve quality of service.

Expanding Ring Search is used for making the route discovery process more efficient [5]. Whenever a node requests a route TTL value is set with RREQ message to be just large enough so that message reaches the destination. When a node initiates a route request it first broadcasts a route request message with small TTL value i.e. TTL start. If the originating node does not receive a RREP message within certain period of time, it rebroadcasts RREQ message with incremented TTL value. The node continues to broadcast messages until it receives a route reply [6]. If the TTL values 
in the route request have reached a certain threshold and still no RREP messages have been received then a network wide flooding is initiated [7].

In AODV routing protocol, an ERS scheme is implemented to start with TTL start and to increase the TTL by TTL increment after each failure. TTL value is generally defined in route request messages which specify the number of times a particular route request message may be re-broadcast [8].

\section{EXPERIMEMTAL METHODOLOGY}

In this experiment, we have generated mobile ad hoc network consisting of 50,100,150,200,250 nodes randomly placed in 500 X500 square meter area and simulation duration is 100 seconds. The simulation tool used for analysis is OPNET 14.5 which is highly preferred by researchers [9].It is a synthetic simulation tool to simulate and analyze for network communication and it can provide three tier model, where the top layer is the network layer that reflects the topology of the network, the middle layer is the node layer that is composed of the corresponding protocol models and the bottom layer is the process model that is described by finite state machines.

Depending on total no. of nodes, size of network and diameter of network D, TTL values for expanding ring search are chosen as given in following Table1. For ERS1, ERS2, ERS3 TTL start and TTL increment are constant and TTL threshold is variable to evaluate the effect of early or delay in network flooding.

Table 1. TTL Parameters

\begin{tabular}{|l|l|l|l|}
\hline ERS & $\begin{array}{l}\text { TTL } \\
\text { start }\end{array}$ & $\begin{array}{l}\text { TTL } \\
\text { increment }\end{array}$ & $\begin{array}{l}\text { TTL } \\
\text { threshold }\end{array}$ \\
\hline ERS1 & 1 & 1 & 6 \\
\hline ERS2 & 1 & 1 & 11 \\
\hline ERS3 & 1 & 1 & 16 \\
\hline
\end{tabular}

The simulation parameters used in experimental scenarios are shown in table 2.

Table 2. Simulation Parameters

\begin{tabular}{|l|l|}
\hline Parameters & Value \\
\hline Simulation Area & $500 \mathrm{~m}$ X 500m \\
\hline Simulation Time & $100 \mathrm{sec}$ \\
\hline Mobile Node Placement & Random Way Point \\
\hline Buffer Size (BS) & 128000 \\
\hline Packet Size & $4096 \mathrm{bits}$ \\
\hline Routing Protocol & AODV \\
\hline No. of Mobile Nodes(MN) & $50 / 100 / 150 / 200 / 250$ \\
\hline Transmit Power & $0.001 \mathrm{~W}$ \\
\hline Data rate & $12 \mathrm{Mbps}$ \\
\hline Node Speed & $2 / 4 / 8 / 16 \mathrm{~m} / \mathrm{s}$ \\
\hline Network Diameter & 35 \\
\hline Node Traversal Time & $0.06 \mathrm{sec}$ \\
\hline
\end{tabular}

The following metrics are evaluated for AODV routing protocol:

Delay: It represents end-to-end delay which refers to the time taken for a packet to be transmitted across a network from source to destination.

Packet Delivery Ratio: It is the ratio of the number of received packets at the destination to the number of packets that are sent by the source node.

Route Discovery Time: The time taken from when a RREQ is assembled until the RREP is received. Number of Hops per Route: It represents no. of hops in each route to every destination in the route of all nodes in the network.

Total Route Requests Sent: It represents the total number of route requests packets sent by all node in the network during route discovery.

Total Replies Sent from Destination: It represents total number of route reply packets sent from all nodes in the network if they are destinations of route requests.

\section{RESULTS AND DISCUSSIONS}

In this section, simulation results have been discussed. The performance of route discovery process is analysed in terms of Delay, Packet Delivery Ratio, Route Discovery Time and Number of Hops per Route, Total Route Request Sent and Total Replies sent from Destination by evaluating effect of early or delay in network flooding and by studying effects of node density and node speed on best chosen TTL value as a basis for expanding ring search.

\subsection{Effect of early or delay in network flooding}

In this section, the performance of route discovery process is analyzed through different TTL threshold values as basis for expanding ring search.

\subsubsection{Delay}

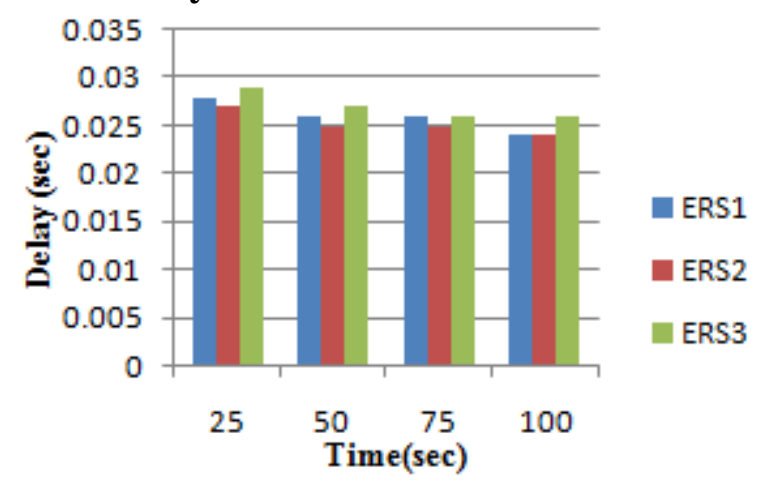

Fig 1: Delay

Fig 1 shows effect of TTL threshold on delay. From figure it is observed that ERS2 attains lowest routing latency which results in quick searching of routes as number of route request propagating are less in ERS2 than ERS3 and ERS1 and results in early route discovery for network defined whereas ERS3 produces largest delay. ERS2 results with less delay as it has less number of route requests propagating per unit time. 


\subsubsection{Packet Delivery Ratio}

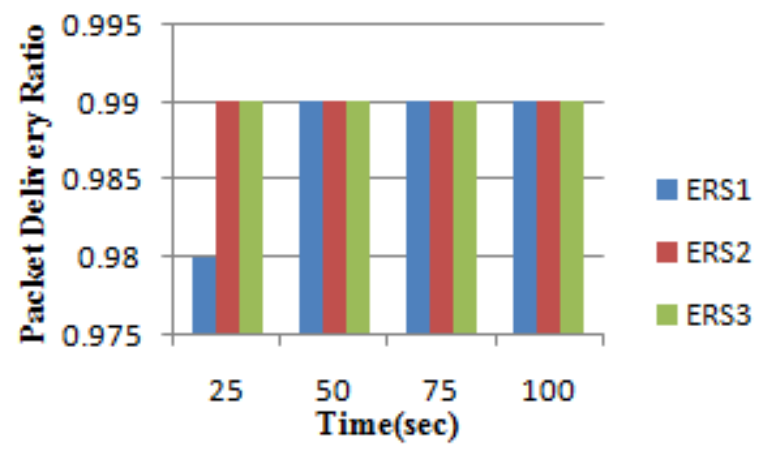

Fig 2: Packet Delivery Ratio

Fig 2 illustrates the packet delivery ratio of expanding ring search with different TTL values. PDR of expanding ring search is nearly 0.99 for all TTL values (ERS1, ERS2 and ERS3) defined.

\subsubsection{Number of Hops per Route}

Number of hops per route is shown in figure 3. Average number of hops per route experienced is less in AODV ERS1 with less TTL threshold as the distance between source and destination for all nodes in the network would be less with less threshold value.

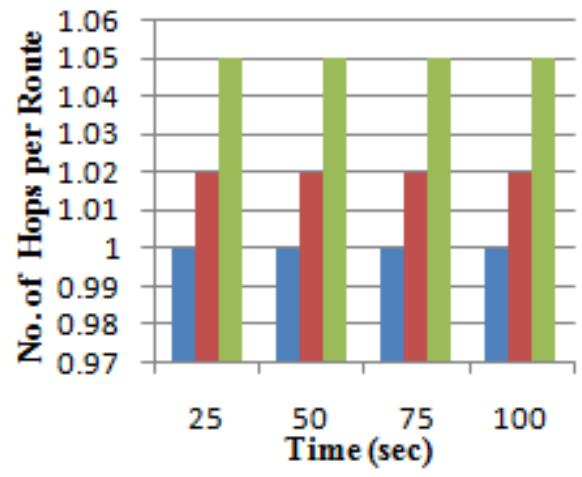

Fig 3: Number of hops per route

\subsubsection{Route Discovery Time}

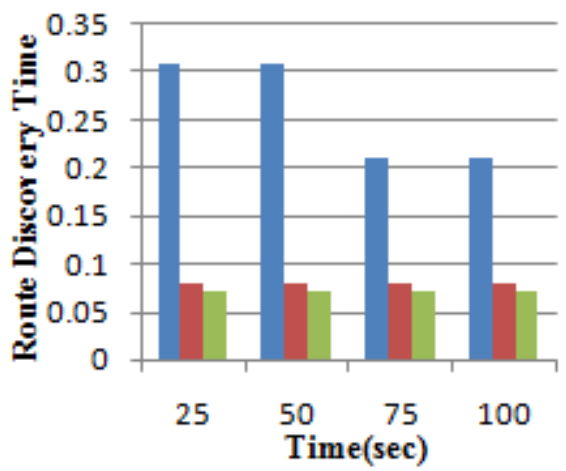

Fig 4: Route Discovery Time
Fig 4 shows effect of TTL threshold on the route discovery time through expanding ring search. From fig it is observed that ERS3 tends to find the earliest path from the source node and destination node.ERS1 takes maximum route discovery time and tends to decrease with time.

\subsubsection{Total Route Requests Sent}

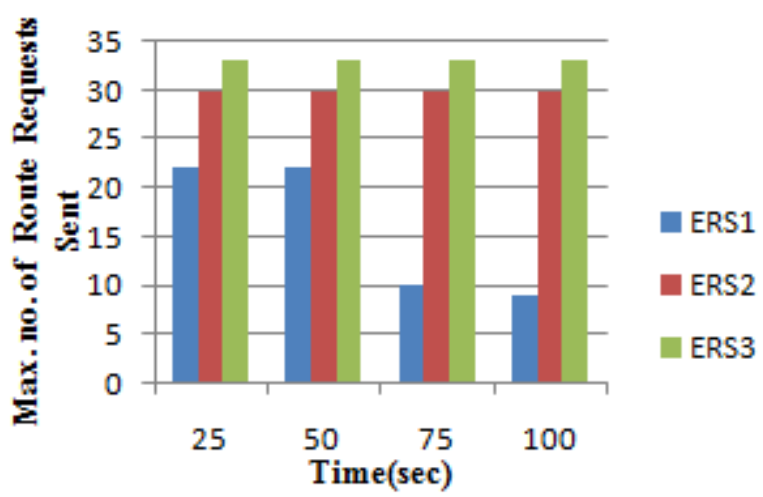

Total route requests sent are observed in fig 5. Reduced number of RREQ is being transmitted by the nodes in ERS1 whereas ERS3 transmit more RREQ by the nodes. This is because of formation of more stable routes and active routes are less prone to route breakage in ERS1. Hence the numbers of control packets transmitted are least in ERS1.

\subsubsection{Total Route Replies Sent from Destination}

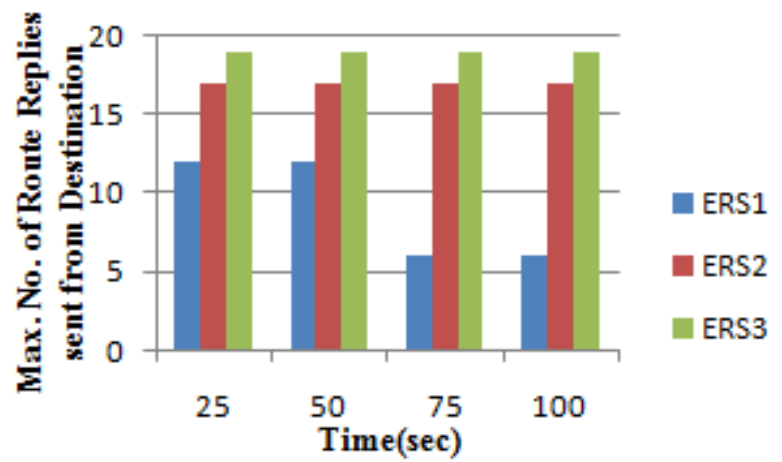

Fig 6: Total Route Replies Sent from Destination

From fig 6 it is clear that ERS3 consistently have more route replies sent from destination than ERS1 and ERS2 which is due to less probability of route errors and packets dropped in ERS3. As ERS1 produces least no. of route request packet sent so it has less number of route replies sent from destination. Fig shows linear increase in ERS1, ERS2 and ERS3 in case of maximum number of route replies sent from destination. 


\subsection{Effect of node density with respect to node speed on expanding Ring search}

In this section, effect of node density with respect to node speed on expanding ring search (ERS1) has been simulated. As ERS1 implemented in route discovery is better in varying node density and node speed environment. Simulations have been done for different number of nodes and for low and high mobility scenarios

\subsubsection{Delay}

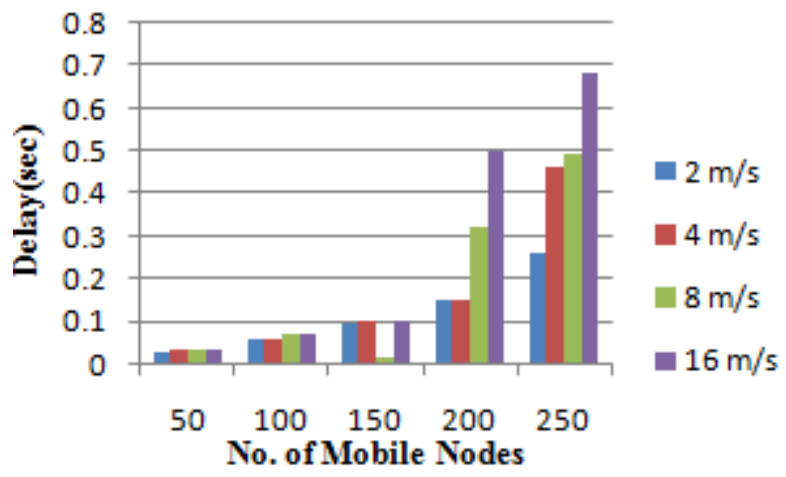

Fig 7: Delay- Varying nodes and node speed

Fig 7 shows delay with varying number of nodes and node speed. This metric is calculated by subtracting time at which first packet was transmitted by source from time at which the first data packet arrived to destination. Delay observed is least for least number of nodes and highest when number of nodes increases to 250. With increase in node speed there are significant changes observed as shown in figure. Linear increase is observed in delay as number of nodes is increasing irrespective of their speed.

\subsubsection{Packet Delivery Ratio}

Graphs show the fraction of data packets that are successfully delivered during simulation node speed versus number of nodes in fig 8. PDR is reducing regularly as no. of nodes are increasing irrespective of their speed as there is no significant effect observed on increasing node speed. Acc to given network parameters, PDR for least number of nodes versus node speed is better amongst all.

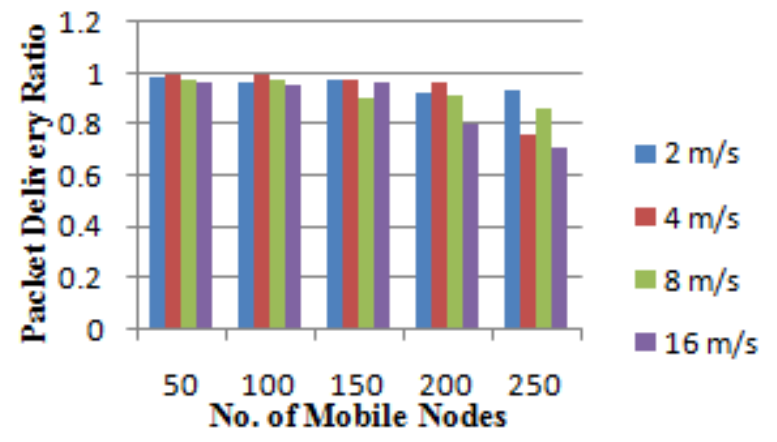

Fig 8: PDR- Varying nodes and node speed

\subsubsection{Number of Hops per Route}

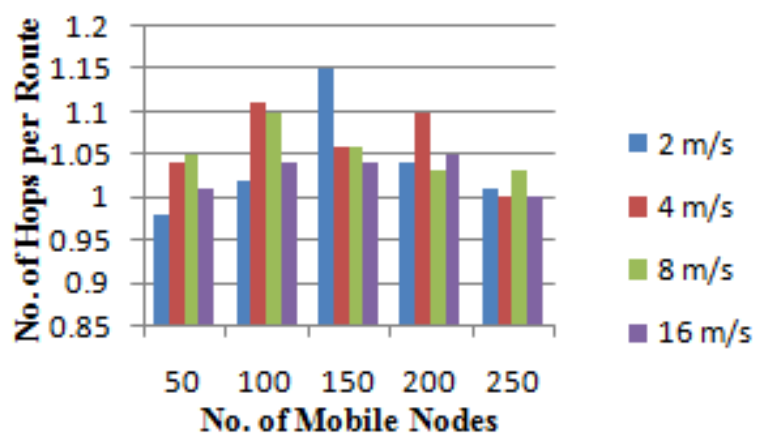

Fig 9: Hops per route- Varying nodes and node speed

Fig 9 shows number of hops per route with increasing number of nodes and node speed. The graph shows that all number of nodes behave differently with increase in mobility of nodes. Least number of hops per route is experienced with least number of nodes and least node mobility.

\subsubsection{Route Discovery Time}

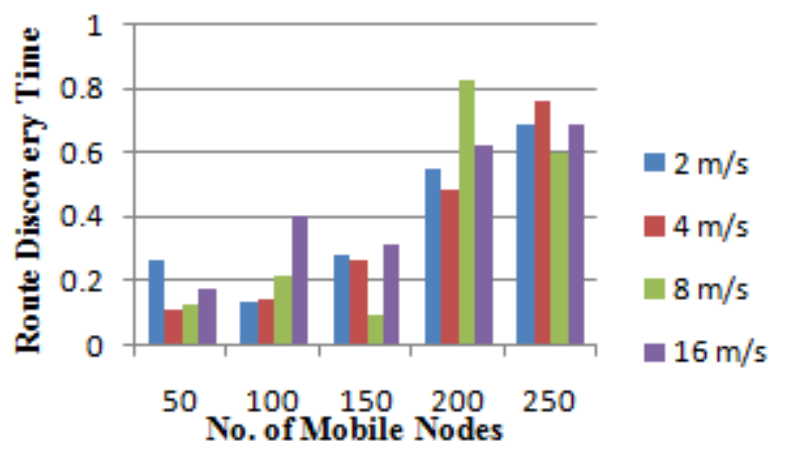

Fig 10: RDT- Varying nodes and node speed

Above graph shows variation of the route discovery time in fig 10 . There is comparable increase in route discovery time as there is increase in node density and node mobility. Less time to discover route is taken by least number of nodes which is due to high probability to discover destination quickly and highest route discovery time is experienced with maximum number of mobile nodes.

\subsubsection{Total Route Requests Sent}

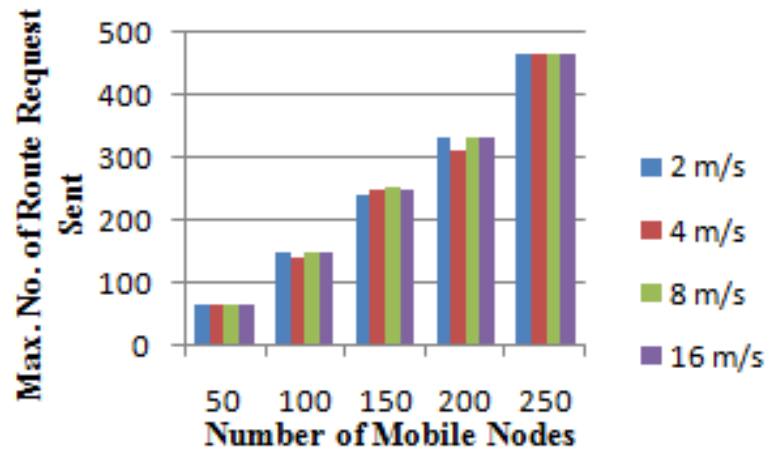

Fig 11: Route Requests- Varying nodes and node speed

With the increase in number of nodes and node mobility, linear increase is observed in total route request sent by all nodes in the network as shown in fig 11 . When nodes 
broadcast RREQ messages those messages are received by more nodes and each node receives a greater number of RREQ messages. There is no effect of speed observed in route request messages sent.

\subsubsection{Total Route Replies Sent from Destination}

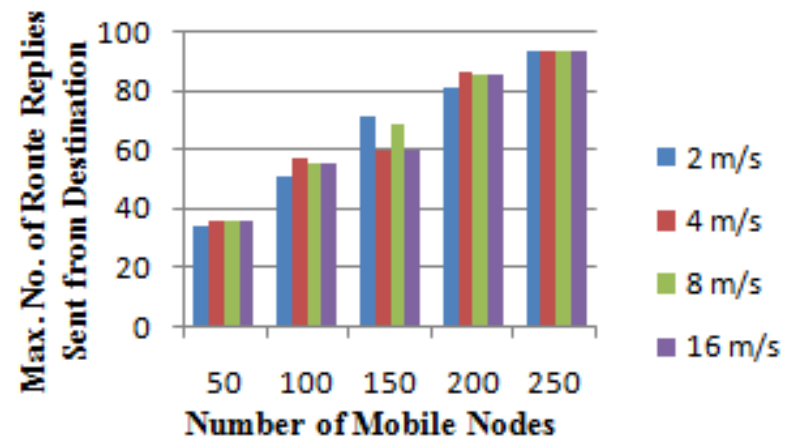

Fig 12: Route Replies-Varying nodes and node speed

Fig 12 shows total route replies sent from destination. As Route requests propagating increases as number of nodes and node mobility increases, total route replies sent from destination also increases with increase in node density and node mobility.

\section{CONCLUSION}

This paper presented expanding ring search proposed for improved quality of service and reliable route discovery. In this paper, performance of route discovery through expanding ring search based TTL parameters is analysed. By camparing ERS1,ERS2 and ERS3 on the basis of various performance metrics it is concluded that ERS1 based TTL is better than ERS2 and ERS3. Based on ERS1 significant and comparable changes are observed on increasing number of nodes but there is no comparable change in simulation results by increasing node speed.

\section{REFERENCES}

[1] Parulpreet Singh, Ekta Barkhodia and gurleen kaur walia,"Evaluation of various Traffic loads in MANET with DSR routing protocol through use of OPNET Simulator", IJDPS,Vol.3 No.3, pp 75-83, May 2012

[2] Raffaele Bruno, Marco Conti and Enrico Grigori, "Mesh Networks: Commodity Multihop Ad hoc Networks", IEEE Communications Magazine, March 2005, pp 123131.

[3] G.S.Mamatha and Dr. S.C. Sharma," A Highly Secured Approach against Attacks in MANETS", International Journal of Computer Theory and Engineering", Vol. 2, No. 5, octuber 2010, pp 816-819

[4] Humaira Nishat, Vamsi Krishna, Dr. D. Srinivasa Rao and shakeel Ahmed," Performance Evaluation of on Demand Routing Protocols AODV and Modified AODV (R-AODV) in MANETS", International Journal of Distributed and Parallel Systems, Vol. 2, No. 1, January 2011

[5] Purvi N.Raanuj and Hiteishi M. Diwanji,"Enhanced Expanding Ring Search Algorithm for AODV", IJCA, Jan 2012

[6] Shweta Mishra, Jyoti Singh, Arti Dixit and Shiva Parkash, "Modified Expanding Ring Search Algorithm for Ad hoc Networks", International Journal of Computer Science and Information Technologies, Vol. 3(3), 2012, pp 4067-4070

[7] Huda Al Amri,Mehran Abolhasan and Tadeusz Wysocki,"Scalability of MANET routing protocols for heterogeneous and homogeneous networks", Elsevier 2010

[8] Amit N. Thakare and Mrs. M. Y. Joshi," performance analysis of AODV and DSR Routing Protocol in Mobile Ad hoc Networks", IJCA, 2010

[9] Lei Guo, Yuhuai Peng, Xingwei Wang, Dingde Jiang and Yinpeng $\mathrm{Yu}$, "Performance evaluation for on-demand routing protocols based on OPNET modules in Wireless Mesh Networks", Computers and Electrical Engineering ,pp 106-114,2011 